VALUE BASED OPHTHALMOLOGY

\title{
Cost utility of photodynamic therapy for predominantly classic neovascular age related macular degeneration
}

\author{
C Hopley, G Salkeld, P Mitchell
}

Br J Ophthalmol 2004;88:982-987. doi: 10.1136/bjo.2003.039131

Series editors: Melissa and Gary Brown

See end of article for authors' affiliations

.....................

Correspondence to: Professor Paul Mitchell, University of Sydney

Department of

Ophthalmology,

Westmead Hospital,

Hawkesbury Road,

Westmead, NSW,

Australia, 2145 ;

paul_mitchell@wmi.usyd.

eduau

Accepted for publication 29 February 2004
Background/aim: Age related macular degeneration (AMD) is the leading cause of severe vision impairment and blindness in older people throughout the developed world and currently affects around 420000 UK citizens. Choroidal neovascularisation (CNV) is treatable with photodynamic therapy (PDT) but is expensive at over $£ 1200$ per treatment. The aim of this study was to assess the cost utility of PDT for better eye, predominantly classic, subfoveal choroidal neovascular lesions secondary to AMD.

Methods: Cost utility analysis (CUA) was conducted to estimate the cost effectiveness of PDT for scenarios involving reasonable $(6 / 12)$ and poor $(6 / 60)$ visual acuity. The models incorporated data from the Treatment of Age-related Macular Degeneration with PDT (TAP) Study and patient based utilities. The incremental CUA was based on decision analytical models, comparing treatment to a placebo comparator. Extensive one way sensitivity analysis of parameters was conducted to determine the robustness of the model. A discount rate of $6 \%$ was used for costs and quality adjusted life years (QALY). Results: Model 1: in people with reasonable initial visual acuity, the cost utility of treating applicable neovascular AMD lesions was £31 607 per QALY saved, with a sensitivity analysis range from £25 285 to $£ 37$ 928. Model 2: in people with poor initial visual acuity, the cost utility was $£ 63214$ per QALY saved, with a sensitivity analysis range from $£ 54183$ to $£ 75856$.

Conclusions: PDT treatment is the only available treatment for some forms of neovascular ("wet") AMD. Under these assumptions, PDT can be considered moderately cost effective for those with reasonable visual acuity but less cost effective for those with initial poor visual acuity. These findings have implications for ophthalmic practice and healthcare planning.
A ge related macular degeneration (AMD) is the major cause of severe visual impairment affecting older people in the developed world. ${ }^{1}$ There are currently around 420000 older people in the United Kingdom with AMD, of which 214000 have registrable blindness secondary to AMD. ${ }^{2}$ People with vision impairment utilise considerable healthcare resources ${ }^{3}{ }^{4}$ and frequently use community support services to maintain independent living in the community. ${ }^{5}$

Late AMD presents in two forms: atrophic ("dry") AMD, which usually has a slow onset and rate of progression; and neovascular ("wet") AMD, which is the more virulent form of the disease and is twice as frequent. ${ }^{6}$ In neovascular AMD, visual deterioration usually results from choroidal neovascularisation $(\mathrm{CNV})$ at the macula, where new vessels develop and evolve to subretinal fibrosis. ${ }^{7} \mathrm{CNV}$ is traditionally classified according to: (1) position relative to the fovea: extrafoveal, juxtafoveal, and subfoveal, and (2) fluorescein angiographic appearance: "classic", "occult," and "mixed."

There are limited options for the treatment of neovascular AMD. Laser photocoagulation has proved to be effective at treating most forms of CNV, but can only be used effectively when the CNV is extrafoveal or juxtafoveal in position. It is generally not helpful when the CNV is subfoveal (as the laser energy would destroy the central macula). Although laser photocoagulation is cost effective, ${ }^{8}$ it can only be used in approximately $15 \%$ of neovascular AMD cases. ${ }^{9}$

Photodynamic therapy (PDT) is a treatment for neovascular AMD using a photosensitising dye (verteporfin) in conjunction with low power diode laser light. In the Treatment of Age-related Macular Degeneration with PDT (TAP) Study, ${ }^{10}$ PDT was shown to be clinically effective in decreasing the risk of vision loss ( $>3$ lines) for people with predominantly classic CNV lesions. PDT using verteporfin, however, is currently relatively expensive at around $£ 1284$ (A \$2670) per treatment.

PDT is currently the only proved therapy for predominantly classic subfoveal neovascular AMD. It has recently completed a lengthy appraisal process and is now funded by the NHS. Other studies have shown PDT to have at best moderate cost effectiveness. ${ }^{11}{ }^{12}$

The aim of this study was to model the cost per quality life year saved (QALY) through the use of PDT treatment, in comparison with placebo treatment, and to refine cost effectiveness estimates for the purpose of funding decisions. The study was conducted from a third party payer perspective.

\section{METHODS}

Cost utility models were created for the following case scenarios:

Model 1

- Reasonable initial visual acuity (VA) 6/12 in the better seeing eye (approximate visual acuity of one mode of the TAP study ${ }^{10}$; this VA level was used in base case 1 of the Sharma models $)^{11}$

- Predominantly classic CNV in that eye (PDT treatment has proved efficacy $)^{10}$

- Poorer vision in the fellow eye (worse than 6/24) ${ }^{11}$

- Average age 75 years (TAP study average). ${ }^{10}$

Abbreviations: $A M D$, age related macular degeneration; $C N V$, choroidal neovascularisation; CUA, cost utility analysis; NICE, National Institute for Clinical Evaluation; PDT, photodynamic therapy; PPP, purchasing power parity; QALYs, quality adjusted life years; VA, visual acuity 
Model 2

- Poor initial VA 6/60 in the better seeing eye (approximate visual acuity of one mode of the TAP study ${ }^{10}$; this VA level was used in base case 2 of the Sharma models) $)^{11}$

- Predominantly classic CNV in that eye (PDT treatment has proved efficacy $)^{10}$

- Poorer vision in the fellow eye (counting fingers and worse $)^{11}$

- Average age 75 years (TAP study average). ${ }^{10}$

The models incorporated data from the TAP study regarding therapeutic efficacy, Australian life tables regarding expected mortality rates, Blue Mountains Eye Study data regarding the increased mortality associated with AMD, and an AMD related utility valuation. Costs and QALYs were adjusted to the reference year of 2003 using a discount rate of $6 \%$.

\section{TAP study findings}

The TAP study ${ }^{10}$ was a double masked clinical study comparing PDT treatment for neovascular AMD, with placebo, and has now published data over 3 years. PDT was shown to decrease the absolute risk of developing 3 lines or more vision loss with PDT treatment by $28 \% \quad(68.7 \%$ developed $>3$ lines vision loss on placebo compared with $40.9 \%$ on PDT treatment) on predominantly classic CNV lesions over 2 years. This difference was statistically significant $(p<0.001)$. The open label TAP extension has indicated minimal worsening of vision for the PDT group in year 3, while using an average of 1.3 PDT treatments per patient over that period..$^{13}$ The average age of subjects was 75 years.

\section{AMD related utility assessment}

The incremental utilities were derived from work of Brown et al. ${ }^{14}$ The measurement of utilities was developed to deal with rational decision making when confronted with uncertainty. ${ }^{15}$ Utilities, by convention, vary from 1.0 (perfect health) to 0.0 (death). Studies have shown that the utilities associated with ophthalmic disease are most highly correlated with visual acuity in the better seeing eye $\mathrm{e}^{14-19}$ and exhibit good retest reliability. ${ }^{20}$ Visual acuity is more important than the pathological cause of eye disease, as far as ophthalmic utility values are concerned. ${ }^{19}$

AMD related utility values have been established, using time trade-off methodology, for different ranges of best corrected visual acuity in the better eye ${ }^{14}$ (table 1). The incremental decrease in visual acuity ( $>3$ lines), associated with the placebo arm, permits an annual calculation of utilities, and hence QALYs, that accrue because of PDT treatment. We assumed a binary state for the TAP study related visual outcomes: the progression or non-progression of 3 line loss of vision related to the placebo and treatment groups. The incremental utilities associated with these

Table 1 Patient based mean utility values associated with better eye visual acuity in people with age related macular degeneration (from Brown et al) $)^{14}$

\begin{tabular}{lll}
\hline Average visual acuity & Utility & $95 \% \mathrm{Cl}$ \\
\hline Initial visual acuity 6/12 (model 1) & 0.81 & $(0.73$ to 0.89$)$ \\
Loss of 3 lines of visual acuity & 0.57 & $(0.47$ to 0.67$)$ \\
Initial visual acuity 6/60 (model 2) & 0.52 & $(0.38$ to 0.66$)$ \\
Loss of 3 lines of visual acuity & 0.40 & $(0.29$ to 0.50$)$ \\
\hline
\end{tabular}

outcomes are 0.24 for people with initial VA 6/12 and 0.12 for people with initial VA $6 / 60^{14}$ (table 1 ).

\section{Cost identification data}

Costs were identified by reviewing the literature, supplemented by expert opinion of AMD resource use, and were measured according to yearly use per affected person. The study included only relevant variable incremental costs (table 2). Schedule fees were obtained from published 2003 Australian Medicare Benefits Schedule (MBS) data (www.health.gov.au/pubs/mbs/index.htm). The MBS is a schedule of fees upon which the government reimburses the providers of medical services. Various other costs such as capital expenditure, overheads costs, and certain labour costs were assumed to be equivalent in both the treatment and placebo arms and were not examined. Indirect costs were also not assessed.

\section{Other data sources and assumptions}

Survival data for the cohort were calculated from ABS life tables. ${ }^{21}$ These rates were adjusted for sex and the increased mortality associated with AMD (risk ratio 1.7), ${ }^{22}$ resulting in a weighted average expected lifespan of 7 years for the average TAP patient (aged 75 years). Australian Federal Government funding for PDT was assumed to be extended over the 7 years modelled. No disutility associated with PDT was modelled owing to the lack of patient based utility values.

\section{Decision analysis and cost utility modelling}

Decision analysis was conducted to determine the incremental utilities associated with PDT treatment (tables 3 and 4). Cost utility analysis was then conducted over a modelled time frame of 7 years. The two alternatives considered in the models were treatment with PDT and placebo treatment. Annual incremental utility values (and hence QALYs) were calculated separately from the associated incremental costs and thereafter combined to derive cost effectiveness ratios.

\section{Model assumptions}

The baseline visual acuity was 6/12 in model 1 and 6/60 in model 2 . The treatment group (in both models) was modelled to receive the following PDT treatments per year (as per the TAP study ${ }^{10}$ ): 3.4 in year $1,2.2$ in year 2 , and 1.3 in year 3 (as per TAP extension study). ${ }^{13}$ The decrease in absolute risk $(28 \%)$ of having a 3 line diminution in vision associated with treatment, was used to finalise the incremental annual utility values. We assumed that to maintain a constant decreased risk at $28 \%$, repeat treatments averaging 1.0 per annum would be necessary over the patient's remaining lifespan.

\section{Purchasing power parity}

Purchasing power parity (PPP) rates were used to convert costs between currencies. Under PPP, exchange rates are adjusted to eliminate the differences in price levels between countries and to better reflect the real value of money when converting the costs of goods and services from one currency to another (www.pacific.commerce.ubc.ca/xr/PPP.html). We used Organisation for Economic Co-operation and Development (OECD) rates (www.oecd.org/std/ppp) to respectively derive the 0.481 and 0.654 factors used to convert Australian dollars and US dollars to pounds sterling in the reference year of 2003.

\section{Discounting}

A real discount rate of 6\% (UK Treasury rate) was used for both costs and QALYs, as recommended by Drummond et al. ${ }^{15}$ 
Table 2 Unit costs for photodynamic therapy with verteporfin using Australian Medicare Benefits Schedule (MBS) item numbers

\begin{tabular}{llrr} 
& & \multicolumn{2}{l}{ Unit cost } \\
\cline { 3 - 4 } Photodynamic therapy & & AS & $£$ \\
\hline Initial consultation & MBS 104 & 69 & 33 \\
Fluorescein angiogram & MBS 11212 and 11215 & 166 & 80 \\
Verteporfin 15 mg vial & Federal Health budget & 2100 & 1010 \\
Procedure & MBS 42884 & 360 & 173 \\
Subsequent consultations & MBS 105 & 35 & 17 \\
Total for 1 treatment & & 2670 & 1284 \\
\hline
\end{tabular}

\section{Sensitivity analysis}

A preliminary analysis of the models was conducted for the relevant variables, using a fixed percentage change in input values. The variables that most affected the incremental cost effectiveness ratios (cost per QALY) were selected for further evaluation in a one way sensitivity analysis. A range of values reflecting the inherent uncertainty for each selected variable was tested.

\section{RESULTS}

\section{Model 1}

This is based on initial VA of 6/12 in the better seeing eye, the development of subfoveal predominantly classic CNV, and receiving treatment annually for a total of 7 years (versus placebo). This resulted in an incremental adjusted QALY of 0.395 for a modelled total of 10.9 PDT treatments. The total adjusted cost associated with this utility equalled £12 478, which results in a cost per QALY of $£ 31607$.

Likely variability or suggested fixed values were tested in the one way sensitivity analysis. This resulted in a range of incremental cost effectiveness ratios from $£ 25285$ to $£ 37928$ per QALY. Lowest and highest cost utility ratios were obtained with incremental utility values of 0.3 and 0.2 respectively. Overall, the sensitivity analysis confirmed the model to be robust (fig 1 ).

\section{Model 2}

This is based on initial VA of 6/60 in the better seeing eye, the development of subfoveal predominantly classic CNV, and receiving treatment annually for a total of 7 years (versus placebo). This resulted in an incremental adjusted QALY of 0.197 for a modelled total of 10.9 PDT treatments. Cost associated with this utility equalled $£ 12478$, which results in a cost/QALY of $£ 63214$.

Likely variability or suggested fixed values were tested in the one way sensitivity analysis. This resulted in a range of incremental cost effectiveness ratios from $£ 54183$ to $£ 75856$ per QALY. Lowest and highest cost utility ratios were obtained with incremental utility values of 0.14 and 0.10 , respectively. Overall, the sensitivity analysis confirmed the model to be robust (fig 2).

\section{DISCUSSION}

AMD is the leading cause of severe visual impairment in the developed world ${ }^{1}$ and it imparts a significant burden on society. Unpublished data suggest an average annual cost of around A\$290 million ( $£ 140$ million) in Australia, with less than a quarter of the total being immediately attributable to direct clinical costs. There are limited options for decreasing this burden on a society-wide scale. Smoking is the most important modifiable risk factor, with current smokers developing AMD three to four times more frequently and 10 years earlier than non-smokers. ${ }^{23}{ }^{24}$ The Age Related Eye Disease Study (AREDS) showed that early AMD could be retarded through the use of high dose zinc and antioxidants. ${ }^{25}$ We have recently shown that a screening and prophylactic treatment programme using zinc and antioxidants is cost effective. ${ }^{26}$

Once late AMD has developed, conventional laser and PDT are currently the only proved treatments. PDT is funded and available in Australia, and has recently received NHS funding in the United Kingdom. Our cost utility model 1 scenario, assuming a baseline VA of 6/12 and predominantly classic CNV in the better seeing eye requiring repeated PDT treatments, would cost a third party payer around £31 607 per QALY. Model 2, assuming a baseline VA of 6/60 and predominantly classic CNV in the better seeing eye requiring repeated PDT treatments, would cost around £63 214 per QALY. Although there are no absolute standards for cost effectiveness, Canadian (and de facto American) guidelines proposed by Laupacis et a ${ }^{27}$ put the model 1 outcome into the moderate cost effectiveness category and the model 2 outcome close to the cost ineffective category.

By comparison, the Sharma (US) 2 model $^{11}$ found a cost per QALY of $£ 56716$ and the Meads (UK) l year model ${ }^{12}$ found a cost per QALY of $£ 137138$ excluding the savings from reduced levels of vision impairment (table 5). Our models differ from the other published models in three key areas.

(1) We assumed that the difference in visual acuity between treatment and placebo groups can be maintained over the affected individuals' expected lifespan by continuing PDT treatments where necessary. The TAP extension data ${ }^{13}$

Table 3 Expected value for photodynamic therapy (PDT) with verteporfin versus placebo treatment in those with initial visual acuity of $6 / 12$

\begin{tabular}{lllll}
\hline & Treatment outcome & Probability & Utility & Result \\
\hline PDT treatment & Stable visual acuity & 0.591 & 0.81 & 0.479 \\
Placebo treatment & Decreased visual acuity & 0.409 & 0.57 & 0.233 \\
Sifference & Stable visual acuity & 0.313 & 0.81 & 0.254 \\
\% difference & Decreased visual acuity & 0.687 & 0.57 & 0.392 \\
\hline
\end{tabular}


Table 4 Expected value for photodynamic therapy (PDT) with verteporfin versus placebo treatment in those with initial visual acuity of $6 / 60$

\begin{tabular}{llllll}
\hline & Treatment outcome & Probability & Utility & Outcome & Total \\
\hline PDT treatment & Stable visual acuity & 0.591 & 0.52 & 0.307 & 0.471 \\
& Decreased visual acuity & 0.409 & 0.4 & 0.164 & 0.163 \\
Placebo treatment & Stable visual acuity & 0.313 & 0.52 & 0.163 & \\
Difference & Decreased visual acuity & 0.687 & 0.4 & 0.275 & \\
\% difference & 0.033 & & & & \\
\hline
\end{tabular}

covering 3 year follow up provide support for this assumption.

(2) We adjusted our mortality rates for the increased mortality associated with visual impairment an AMD, thereby producing a 7 year model.

(3) We did not include any disutility associated with PDT treatment, unlike the Sharma models.

Modelling over a longer time frame does affect the results found. This occurs because the treatment is expensive and the largest costs occur upfront, whereas the benefits occur when visual acuity fails to deteriorate in the future. By only modelling over 1 or 2 years, any benefit (utility or QALY) occurring thereafter is ignored; however, all the associated costs have already been incurred and thus are tallied against the existing, smaller, benefit. In effect by doing so, the modeller is making an implicit assumption that there is no future benefit from the treatment. We now know this is not

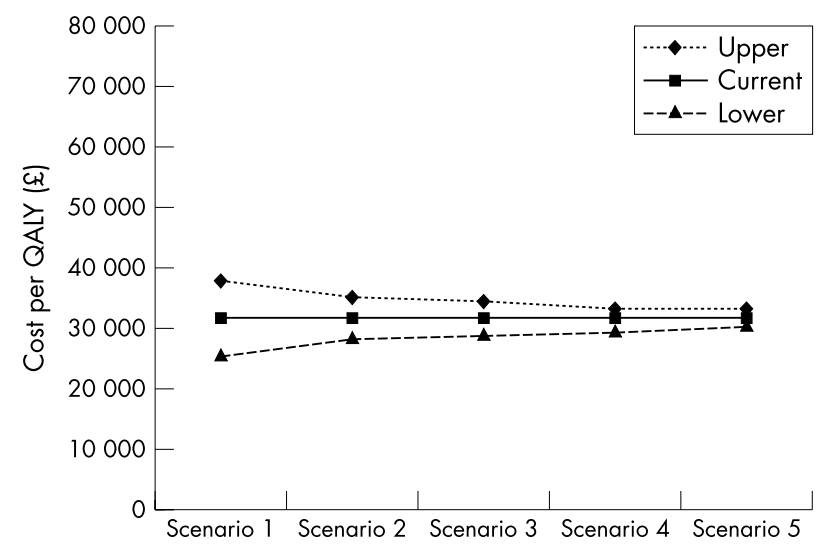

\begin{tabular}{|l|l|c|c|c|}
\hline & Variable & Lower & Current & Upper \\
\hline Scenario 1 & Incremental utility value & 0.30 & 0.24 & 0.20 \\
\hline Scenario 2 & $\%$ Risk reduction & $31 \%$ & $28 \%$ & $25 \%$ \\
\hline Scenario 3 & $\begin{array}{l}\text { Continuing annual PDT } \\
\text { treatments }\end{array}$ & 0.7 & 1.0 & 1.3 \\
\hline Scenario 4 & Discount rate: QALYs ${ }^{\dagger}$ & $3 \%$ & $6 \%$ & $8 \%$ \\
\hline Scenario 5 & Discount rate: costs & $8 \%$ & $6 \%$ & $3 \%$ \\
\hline
\end{tabular}

`Photodynamic therapy

†Quality adjusted life years

Figure 1 Model 1: one way sensitivity analysis of incremental cost effectiveness ratios (cost per QALY) for each scenario. the case. ${ }^{13}$ Sharma et $a l^{11}$ did produce 11 year year cost utility models for PDT but discounted the findings because of the lack of supporting data at that time.

Our study also does not take into account a number of the potential benefits from a reduction in reported impacts on independent living from vision impairment in an older population, as found in various population based studies of eye disease. The Blue Mountains Eye study has reported that corrected visual acuity worse than $6 / 18$ is associated with an eightfold increased risk of hip fracture and a $27 \%$ attributable risk for hip fracture from vision impairment. ${ }^{28}$ Vision impairment of 6/12 and worse is associated with a threefold increase in the use of health and community care services (for example, meals on wheels, home help), ${ }^{5}$ a 1.8 -fold increase in admissions to nursing homes, ${ }^{29}$ together with impaired self rated health ${ }^{30}$ and health related quality of life. ${ }^{31}$

A recent TAP report $^{32}$ demonstrated that verteporfin therapy reduced the risk of a clinically relevant loss of

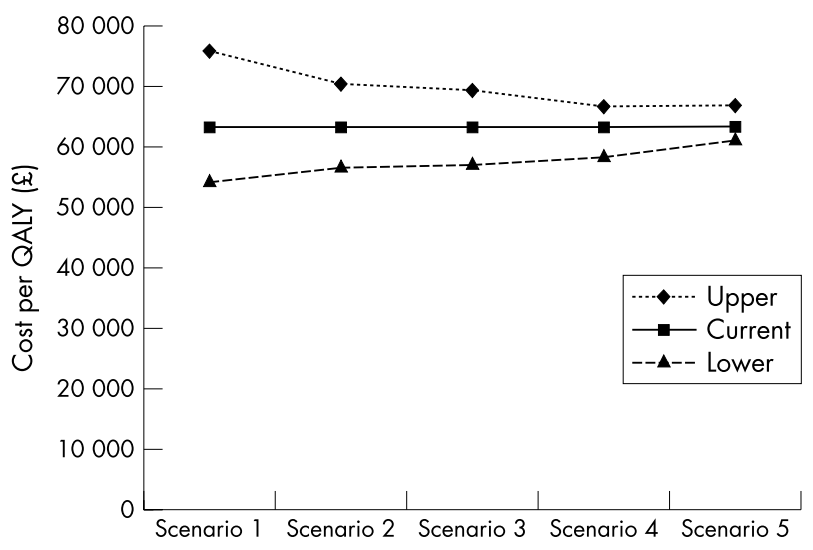

\begin{tabular}{|l|l|c|c|c|}
\hline & Variable & Lower & Current & Upper \\
\hline Scenario 1 & Incremental utility value & 0.10 & 0.12 & 0.14 \\
\hline Scenario 2 & $\%$ Risk reduction & $31 \%$ & $28 \%$ & $25 \%$ \\
\hline Scenario 3 & $\begin{array}{l}\text { Continuing annual PDT } \\
\text { treatments }\end{array}$ & 0.7 & 1.0 & 1.3 \\
\hline Scenario 4 & Discount rate: QALYs ${ }^{\dagger}$ & $3 \%$ & $6 \%$ & $8 \%$ \\
\hline Scenario 5 & Discount rate: costs & $8 \%$ & $6 \%$ & $3 \%$ \\
\hline
\end{tabular}

`Photodynamic therapy

${ }^{\dagger}$ Quality adjusted life years

Figure 2 Model 2: one way sensitivity analysis of incremental cost effectiveness ratios (cost per QALY) for each scenario. 
Table 5 Comparison of cost utility output from different published modelling approaches with different visual acuity (VA) starting points

\begin{tabular}{|c|c|c|}
\hline Model & Details & Cost/QALY \\
\hline \multicolumn{3}{|c|}{ Hopley et al (this study) } \\
\hline Model 1 & Reasonable VA, continuous PDT, 7 year model & 31607 \\
\hline Model 2 & Poor VA, continuous PDT, 7 year model & 63214 \\
\hline \multicolumn{3}{|l|}{ Sharma et al ${ }^{11}$} \\
\hline Base case 1 & Reasonable VA, 2 years PDT, 2 year model & 56716 \\
\hline Base case 2 & Poor VA, 2 years PDT, 2 year model & 113786 \\
\hline $\begin{array}{l}\text { Meads et al } \\
\text { Model }\end{array}$ & 1 year model & 137138 \\
\hline
\end{tabular}

contrast sensitivity, with the greatest effect in patients with predominantly classic subfoveal CNV. This could be due to the development of a flatter and smaller scar in treated patients. Given the association between contrast sensitivity and visual disability and its importance as a predictor of falls ${ }^{33}$ and possibly, hip fractures, ${ }^{28}$ the beneficial effects of verteporfin therapy on contrast sensitivity outcomes are likely to also have a favourable impact on patients' daily activities.

Economic modelling is only as good as the underlying data used. The TAP study ${ }^{10}$ is a well conducted, randomised controlled study and can be regarded as level 2 data. The utility assessment has been used in the other cost utility studies and has been peer reviewed. The unit cost data were taken from an Australian setting. The converted PDT cost figure, however, is comparable to published UK figures. ${ }^{12}$ The sensitivity analysis gives a range of effects that enable robust cost generalisation across similar populations of European origin.

It must be borne in mind that model $\mathrm{l}$ is close to being a best case scenario as far as assessing the cost utility of PDT is concerned, as it assumes reasonable vision in the affected and better seeing eye. As ophthalmic utilities are a function of vision in the better seeing eye, if the CNV affected eye was partnered by another good seeing eye, the changes in utility after treatment would be smaller (and hence the treatment less cost effective). The model 2 data are relevant, as funding is available in many countries for patients with initial visual acuity reduced to $6 / 60$. Our analysis indicates a reduced cost utility for this level of initial visual function. Finally, in modelling the repeated use of PDT, we may be overly pessimistic in requiring an average of one treatment per year for years 4-7. If fewer treatments were needed, this would tend to improve the overall cost effectiveness ratios found.

\section{CONCLUSIONS}

AMD is a major problem affecting many older people in the developed world. Preventive measures can be taken for early AMD (smoking cessation and use of zinc/antioxidant supplements). PDT is at least moderately cost effective, at around £31 607 per QALY, in people with reasonable visual acuity given our assumptions (for example, better eye affected, reasonable initial visual acuity, predominantly classic CNV lesion). PDT, however, is relatively cost ineffective in those with poor initial visual acuity, at around £63 214 per QALY. Our estimates may be pessimistic because of assumptions made regarding the need for continuing treatment. Our findings have relevance for health policy decision makers.

\section{Authors' affiliations}

C Hopley, P Mitchell, Centre for Vision Research, Department of Ophthalmology and the Westmead Millennium Institute, the University of Sydney, Australia
G Salkeld, School of Public Health, University of Sydney, Sydney, Australia

This study was supported by an initiating grant from the Westmead Millennium Institute, University of Sydney.

\section{REFERENCES}

1 Wang JJ, Foran S, Mitchell P. Age-specific prevalence and causes of bilateral and unilateral visual impairment in older Australians: the Blue Mountains Eye Study. Clin Experiment Ophthalmol 2000;28:268-73.

2 Owen CG, Fletcher AE, Donoghue $M$, et al. How big is the burden of visual loss caused by age related macular degeneration in the United Kingdom? Br J Ophthalmol 2003;87:312-17

3 Wright SE, Keeffe JE, Thies LS. Direct costs of blindness in Australia [in process citation]. Clin Experiment Ophthalmol 2000;28:140-2.

4 Chiang YP, Bassi $\amalg$, Javitt JC. Federal budgetary costs of blindness. Milbank $Q$ 1992;70:319-40.

5 Wang JJ, Mitchell P, Smith W, et al. Impact of visual impairment on use of community support services by elderly persons: the Blue Mountains Eye Study. Invest Ophthalmol Vis Sci 1999;40:12-19.

6 Mitchell P, Smith W, Attebo K, et al. Prevalence of age-related maculopathy in Australia. The Blue Mountains Eye Study. Ophthalmology 1995; 102:1450-60

7 Bressler NM, Bressler SB, Fine SL. Age-related macular degeneration. Surv Ophthalmol 1988;32:375-413.

8 Macular Photocoagulation Study Group. Laser photocoagulation for juxtafoveal choroidal neovascularization. Five-year results from randomised clinical trials. Arch Ophthalmol 1994;112:500-9.

9 Moisseiev J, Alhalel A, Masuri R, et al. The impact of the macular photocoagulation study results on the treatment of exudative age-related macular degeneration. Arch Ophthalmol 1995;113:185-9.

10 Treatment of age-related macular degeneration with photodynamic therapy (TAP) Study Group. Photodynamic therapy of subfoveal choroidal neovascularization in age-related macular degeneration with verteporfin: one-year results of 2 randomised clinical trials-TAP report. Arch Ophthalmol 1999;117:1329-45.

11 Sharma S, Brown GC, Brown MM, et al. The cost-effectiveness of photodynamic therapy for fellow eyes with subfoveal choroidal neovascularization secondary to age-related macular degeneration. Ophthalmology 2001;108:2051-9.

12 Meads C, Salas C, Roberts T, et al. Clinical effectiveness and cost-utility of photodynamic therapy for wet age-related macular degeneration: a systematic review and economic evaluation. Health Technol Assess 2003;7:1-108.

13 Blumenkranz MS, Bressler NM, Bressler SB, et al. Verteporfin therapy for subfoveal choroidal neovascularization in age-related macular degeneration: three-year results of an open-label extension of 2 randomised clinical trials TAP Report no 5. Arch Ophthalmol 2002;120:1307-14.

14 Brown GC, Sharma S, Brown MM, et al. Utility values and age-related macular degeneration. Arch Ophthalmol 2000;118:47-51.

15 Drummond MF, O'Brien B, Stoddart GL, et al. Methods for the economic evaluation of health care programmes, 2nd ed. New York: Oxford University Press, 1997.

16 Brown MM, Brown GC, Sharma S, et al. Quality of life with visual acuity loss from diabetic retinopathy and age-related macular degeneration. Arch Ophthalmol 2002;120:481-4.

17 Brown MM, Brown GC, Sharma S, et al. Health care economic analyses and value-based medicine. Surv Ophthalmol 2003;48:204-23.

18 Sharma S, Oliver-Fernandez A, Bakal J, et al. Utilities associated with diabetic retinopathy: results from a Canadian sample. $\mathrm{Br} J$ Ophthalmol 2003;87:259-61.

19 Brown MM, Brown GC, Sharma S, et al. Utility values associated with blindness in an adult population. Br J Ophthalmol 2001;85:327-31.

20 Brown GC, Brown MM, Sharma S, et al. The reproducibility of ophthalmic utility values. Trans Am Ophthalmol Soc 2001;99:199-203.

21 Australian Bureau of Statistics. Deaths Australia 1996, ABS Catalogue. Canberra: ABS, 1997;3302:0, 62-3.

22 Wang JJ, Mitchell P, Simpson JM, et al. Visual impairment, age-related cataract, and mortality. Arch Ophthalmol 2001;119:1186-90. 
23 Mitchell P, Wang JJ, Smith W et al. Smoking and the 5-year incidence of age-related maculopathy: the Blue Mountains Eye Study. Arch Ophthalmol 2002; 120:1357-63

24 Mitchell P, Chapman S, Smith W. Smoking is a major cause of blindness. Med J Aust 1999;171:173-4.

25 AREDS. A randomised, placebo-controlled, clinical trial of high-dose supplementation with vitamins $C$ and $E$, beta carotene, and zinc for agerelated macular degeneration and vision loss. AREDS report no 8 . Arch Ophthalmol 2001;119:1417-36.

26 Hopley C, Salkeld G, Wang JJ, et al. Cost utility of screening and treatment for early age related macular degeneration with zinc and antioxidants. Br J Ophthalmol 2004;88:450-4.

27 Laupacis A, Feeny D, Detsky AS, et al. How attractive does a new technology have to be to warrant adoption and utilization? Tentative guidelines for using clinical and economic evaluations. CMAJ 1992;146:473-81.
28 Ivers RQ, Optom B, Cumming RG, et al. Visual risk factors for hip fracture in older people. J Am Geriatr Soc 2003;51:356-63.

29 Wang JJ, Mitchell P, Cumming RG, et al. Visual impairment and nursing home placement in older Australians: the Blue Mountains Eye Study. Ophthalmic Epidemiol, 2003 Feb, 10:3-13.

30 Wang JJ, Mitchell P, Smith W. Vision and low self-rated health: the Blue Mountains Eye Study. Invest Ophthalmol Vis Sci 2000;41:49-54.

31 Chia EM, Wang JJ, Rochtchina E, et al. Impact of bilateral visual impairment on health-related quality of life: the Blue Mountains Eye Study. Invest Ophthalmol Vis Sci 2004:45:71-6.

32 Rubin GS, Bressler NM. Effects of verteporfin therapy on contrast on sensitivity: results from the Treatment of Age-Related Macular Degeneration With Photodynamic Therapy (TAP) investigation. TAP report No 4. Retina 2002;22:536-44

33 Lord SR, Clark RD, Webster IW. Physiological factors associated with falls in an elderly population. J Am Geriatr Soc 1991;39:1194-200. 